COMUNICAC̣ÃO BREVE

JOSÉ PAULO FIKS

PATRÍCIA MATTOS

MARIO DINIS MATEUS

ANDREA A. FEIJÓ DE MELLO

MARCELO FEIJÓ DE MELLO
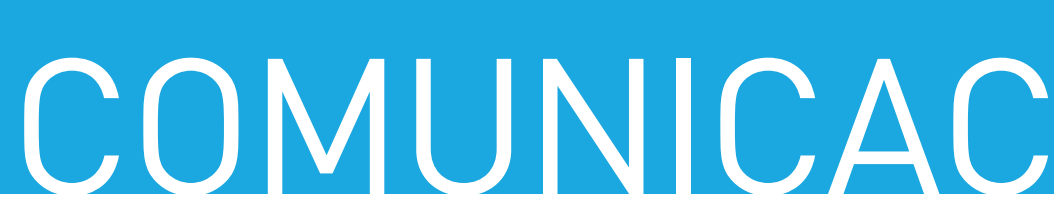

$\tilde{A}$

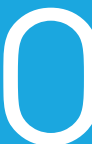

\title{
A CRISE NA SAÚDE MENTAL EM DECORRÊNCIA DA COVID-19: DIRETRIZES PARA UM SUPORTE PSICOLÓGICO EM PANDEMIA POTENCIALMENTE TRAUMÁTICA
}

\section{THE MENTAL HEALTH CRISIS TRIGGERED BY COVID-19: GUIDELINES FOR PROVISION OF PSYCHOLOGICAL SUPPORT DURING A POTENTIALLY TRAUMATIC PANDEMIC}

\section{Resumo}

A pandemia causada pela COVID-19 já é uma catástrofe mundial. Provoca uma crise sem precedentes na história contemporânea, o que também exige uma resposta de clínicos e pesquisadores em saúde mental. Neste artigo, os autores clarificam alguns conceitos do campo de trauma psíquico envolvidos nesse contexto. Em resposta ao impacto psíquico desta pandemia, um tutorial com uma estratégia de suporte psicológico é contemplado, com destaque para a psicopatologia decorrente do estresse e do transtorno de estresse póstraumático (TEPT).]

Palavras-chave: Pandemias, crise em saúde mental, TEPT.

\section{Abstract}

The pandemic caused by COVID-19 is already a worldwide catastrophe. It has caused an unprecedented crisis in contemporary history, which also requires a response from mental health clinicians and researchers. In this article, the authors clarify some concepts in the field of psychological trauma involved in this context. In response to the mental impact of this pandemic, a tutorial including a psychological support strategy is contemplated, with emphasis on the psychopathology resulting from stress and post-traumatic stress disorder (PTSD).

Keywords: Pandemics, mental health crisis, PTSD.
Desde as últimas epidemias, como a da síndrome respiratória aguda grave (SARS) (2002-2003), gripe suína (2009-2010), ebola (2014-2016) e Zika (20152016), havia predições de uma nova pandemia viral com repercussões psíquicas devastadoras generalizadas e em escala global ${ }^{1}$. Esse flagelo chegou com a COVID19, acarretará modificações de grande magnitude para a humanidade e exige intervenções imediatas no campo da saúde mental.

Para delimitações de possíveis transtornos mentais, com imediato estabelecimento de ações preventivas e curativas, são fundamentais clarificações de conceitos no campo do trauma psíquico. Assim, a ideia de crise em saúde mental como um acontecimento inesperado, disruptivo e potencialmente traumático implica a análise do impacto dessa pandemia sobre o psiquismo. Isso deve ser seguido por uma tomada de decisão que considere o problema e suas possíveis soluções, um projeto de implementação e, finalmente, um plano de sustentação por um período de tempo prolongado.

Esta comunicação considera os itens descritos acima e tem como objetivo uma proposta de suporte psicológico sustentável durante esse período de crise em saúde mental, conforme descrito acima.

A atual pandemia causada pela COVID-19 aponta para a consideração de três estados contemplados pela clínica e pesquisa em trauma psíquico: o estresse, a catástrofe e o transtorno do estresse pós-traumático 


\section{COVID-19}

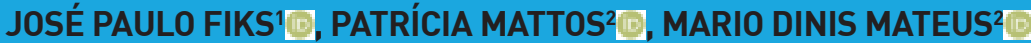 ANDREA A. FEIJÓ DE MELLO3ㅁ, MARCELO FEIJÓ DE MELLO4믐}

Psiquiatra. Pós-Doutor em Ciências da Saúde, Universidade Federal de São Paulo (UNIFESP). São Paulo, SP. Pesquisador, Serviço de Assistência e Pesquisa em Violência e Estresse Pós-Traumático (PROVE), Departamento de Psiquiatria, UNIFESP. Escola Paulista de Medicina (EPM), São Paulo, SP. ${ }^{2}$ Psiquiatra. Doutorlal em Ciências da Saúde, UNIFESP, São Paulo, SP. Pesquisador(a), PROVE, UNIFESP, São Paulo, SP. ${ }^{3}$ Psiquiatra, Pós-Doc em Ciências da Saúde, UNIFESP, São Paulo, SP. Professora, Departamento de Psiquiatria, UNIFESP-EPM, São Paulo, SP. Coordenadora geral, PROVE, UNIFESP, São Paulo, SP. ${ }^{4}$ Psiquiatra. Professor livre-docente, Departamento de Psiquiatria, UNIFESP-EPM, São Paulo, SP. Fundador e docente responsável pelo PROVE, UNIFESP, São Paulo, SP.

(TEPT) complexo². Essa compreensão permite uma ação imediata na prevenção e suporte para os quadros mentais mais comuns nessa crise ${ }^{3}$.

O estresse é um conjunto de respostas fisiológicas que permitem ao organismo recuperar naturalmente sua homeostase frente a um estímulo. Em situações de crise, em que a pressão é constante, a resposta de estresse é contínua, repetida ou crônica. Isso pode exceder as capacidades do organismo de se recuperar e/ou resistir, levando ao esgotamento do indivíduo como um todo. O estresse provoca a tentativa de o organismo voltar à homeostase, mas quando isso é impossível, surge uma exigência biológica conhecida como carga alostática. Esta acarreta alterações neuroendócrinas, epigenéticas e imunológicas de acordo com as características individuais, especialmente em evento estressante prolongado como o da atual pandemia4,5.

Apesar de ainda não ser considerada uma patologia, mas uma apresentação de saúde ocupacional, a síndrome de burnout é associada a situações de estresse crônico, com falha de gerência em local de trabalho, que leva o indivíduo a um colapso. Essa síndrome é caracterizada por três dimensões de comprometimentos: sentimentos de exaustão ou esgotamento de energia; aumento do distanciamento mental e sentimentos negativos ou de insolência sempre relacionados ao trabalho, com redução da eficácia profissional ${ }^{6}$.

Sintomas como irritação, impressão de exaustão, alerta constante, labilidade de humor, insônia, dificuldades com a concentração, dores e tendência ao abuso de substâncias são comuns. Indivíduos submetidos a uma rotina de trabalho estressante durante a pandemia estão mais propícios ao burnout, especialmente profissionais dos serviços essenciais e da saúde?

Catástrofes são eventos naturais de grande extensão que provocam alterações permanentes em uma comunidade, acarretando sofrimento físico e psíquico sobre um grande número de vítimas ${ }^{8}$. A atual pandemia se inscreve na história contemporânea pelo ineditismo do aspecto catastrófico global. A rapidez do contágio, a virulência, o tropismo, especialmente pelas vias pulmonares, com imagem radiológica típica, a letalidade da população mais idosa e o acometimento brutal das equipes de saúde fazem dessa pandemia um acontecimento trágico e único, ainda em evolução desconhecida.

Essa circunstância catastrófica é potencializadora do TEPT em sua forma complexa, pela longa exposição aos efeitos deletérios da COVID-19, ainda sem tratamento eficaz nem prazo para vacinas. Como fatores predisponentes ao TEPT complexo, estão as consequências do confinamento ainda por tempo indeterminado, que pode gerar situações de violência interpessoal, além do abuso de substâncias?.

O desmoronamento do sistema de saúde, os estados de luto e a calamidade econômica que se anuncia também exigem ações imediatas preventivas e curativas em saúde mental ${ }^{10}$. Estas devem ser objetivas e focadas na redução de sintomas de estresse e TEPT complexo, além de fortalecimento emocional e social, buscando um projeto possível de resiliência ${ }^{11}$.

○ suporte psicológico dirigido para a atual pandemia deve considerar quatro campos relativos ao funcionamento psíquico: manutenção do cotidiano, manejo do estresse, enfoque na resiliência e estímulo social $^{12,13}$

Este tutorial é indicado para indivíduos sem história psiquiátrica anterior, bem como pacientes já em tratamento e também para indivíduos envolvidos em vulnerabilidade específica da pandemia, como trabalhadores de serviços essenciais e profissionais da saúde ainda em atividade com risco de burnout.

As quatro ações são descritas abaixo de forma a contemplar o atendimento em saúde mental com foco no suporte psicológico durante a pandemia atual:

1. Manutenção do cotidiano: esse item deve focar em informações objetivas sobre a pandemia e clarificações de dúvidas por parte do terapeuta.

1.1 Estímulo à criação de uma rede da confiança do paciente para abastecimento de atualizações, com limite a noticiários e redes sociais.

1.2. É importante ressaltar a manutenção de atividades básicas, como sono, alimentação, hidratação, lazer, exercícios e introspecção. Estas são garantidoras de um funcionamento psíquico elementar para o enfrentamento da crise. 
2. Manejo do estresse: uma lista objetiva de pontos fortes para encarar a crise, bem como as vulnerabilidades pessoais, deve ser preparada com os seguintes enfoques:

2.1 Quaissão os limites de ação para confronto das adversidades provocadas pela pandemia?

$2.2 \bigcirc$ que mais pode ser explorado como potencial psicológico para o enfrentamento da crise?

2.3 Uma lista de preocupações e análise dos respectivos itens pode ser fundamental no impedimento de pensamentos negativos, que em momentos de estresse invadem o psiquismo.

2.4 Para pacientes em tratamento psiquiátrico, um exame mais cuidadoso das fragilidades percebidas pode evitar recaídas.

3. Enfoque na resiliência: perante uma catástrofe, a enumeração de prováveis modificações no cenário mundial com impacto pessoal (perda de trabalho e adiamento de projetos) deve fazer parte desse item.

3.1 Reconhecimento dos afetos (sentimentos e emoções) decorrentes da pandemia, tais como perdas de entes queridos, instabilidade financeira, limite na locomoção, desabastecimentos e confinamento por tempo indeterminado devem receber atenção especial.

3.2 Estratégias para a elaboração de um novo cotidiano possível e cenários personalizados para o evento mais prolongado devem ser a meta deste item para o fortalecimento psicológico com olhar para um futuro próximo. Assim, a resiliência é entendida aqui como um enfrentamento e fortalecimento da resistência para evitar o trauma psíquico desencadeado pela pandemia.

4. Estímulo social: os três enfoques anteriores se entrelaçam em ações de reforço recíproco, visando a uma repercussão positiva na dimensão social que permeia a crise. Esse item é importante base para a formulação de uma rede social de apoio, visando a um período imediatamente posterior à pandemia ${ }^{14}$.

$4.1 \bigcirc$ conceito de capital social, definido como arranjos tais como redes, algoritmos e confiança social, facilita a organização e a colaboração para o benefício mútuo ${ }^{15}$. Além disso, relaciona-se a melhores desfechos em saúde em geral16.

4.2 engajamento social promove o sentimento de pertencimento, estimula a participação na comunidade, o altruísmo, influencia positivamente na manutenção de um novo cotidiano, reduz o estresse e promove a resiliência.

Este tutorial foi elaborado com base nas publicações mais recentes que abordam o campo da saúde mental e os impactos provocados pela pandemia da COVID-19.

Artigo submetido em 12/04/2020, aceito em 13/04/2020. Os autores informam não haver conflitos de interesse associados à publicação deste artigo.

Fontes de financiamento inexistentes.

Correspondência: José Paulo Fiks, Rua Major Maragliano, 241. CAISM/UNIFESP, Vila Mariana, CEP 04017-030, São Paulo, SP. E-mail:.jpfiks@uol.com.br

\section{Referências}

1. Perrin PC, McCabe OL, Everly GS Jr, Links JM. Preparing for an influenza pandemic: mental health considerations. Prehosp Disaster Med. 2009:24:223-30.

2. Huremović D. Psychiatry of pandemics: a mental health response to infection outbreak. New York: Springer; 2019.

3. American Psychiatric association (APA). APA Coronavirus resources [Internet]. 2020 Mar [cited 2020 Apr 14]. www.psychiatry.org/psychiatrists/ covid-19-coronavirus

4. McEwen BS. Physiology and neurobiology of stress and adaptation: central role of the brain. Physiol Rev. 2007;87:873-904.

5. McEwen BS. Neurobiological and systemic effects of chronic stress. chronic stress. Chronic Stress (Thousand Oaks). 2017 Jan-Dec;1. doi: 


\section{COVID-19}

JOSÉ PAULO FIKS'ㅁ, PATRÍCIA MATTOS 22 , MARIO DINIS MATEUS ${ }^{2}[$,

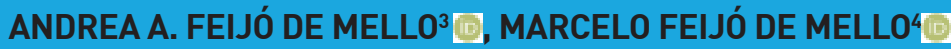

1 Psiquiatra. Pós-Doutor em Ciências da Saúde, Universidade Federal de São Paulo (UNIFESP), São Paulo, SP. Pesquisador, Serviço de Assistência e Pesquisa em Violência e Estresse Pós-Traumático (PROVE), Departamento de Psiquiatria, UNIFESP. Escola Paulista de Medicina (EPM), São Paulo, SP. ${ }^{2}$ Psiquiatra. Doutorlal em Ciências da Saúde, UNIFESP, São Paulo, SP. Pesquisador(a), PROVE, UNIFESP, São Paulo, SP. ${ }^{3}$ Psiquiatra, Pós-Doc em Ciências da Saúde, UNIFESP, São Paulo, SP. Professora, Departamento de Psiquiatria, UNIFESP-EPM, São Paulo, SP. Coordenadora geral, PROVE, UNIFESP, São Paulo, SP. ${ }^{4}$ Psiquiatra. Professor livre-docente, Departamento de Psiquiatria, UNIFESP-EPM, São Paulo, SP. Fundador e docente responsável pelo PROVE, UNIFESP. São Paulo, SP.

10.1177/2470547017692328. Epub 2017 Apr 10.

6. World Health Organization (WHO). International Classification of Diseases 11th Revision [Internet]. 2019 [cited 2020 Mar 14]. icd.who.int/en

7. Fiorillo A, Gorwood P. The consequences of the COVID-19 pandemic on mental health and implications for clinical practice. Eur Psychiatry. 2020;63:e32.

8. Braga LL, Fiks JP, Mari JJ, Mello MF. The importance of the concepts of disaster, catastrophe, violence, trauma and barbarism in defining posttraumatic stress disorder in clinical practice. BMC Psychiatry. 2008;8:68.

9. Karatzias T, Cloitre M, Maercker A, Kazlauskas E, Shevlin M, Hyland P, et al. PTSD and Complex PTSD: ICD-11 updates on concept and measurement in the UK, USA, Germany and Lithuania. Eur J Psychotraumatol. 2018;8(Suppl 7):1418103.

10. Inchausti F, MacBeth A, Hasson-Ohayon I, Dimaggio G. Psychological interventions and the Covid-19 pandemic [Internet]. 2020 Apr 2 [cited 2020 Apr 14]. doi.org/10.31234/osf.io/8svfa

11. Halpern J, Nitza A, Vermeulen K, editors. Disaster mental health case studies. New York: Routledge; 2019 ,

12. Inter-Agency Standing Committee (IASC). IASC Interim Briefing Note on COVID-19 Outbreak
Readiness and Response Operations - MHPSS (Portuguese) [Internet]. $2020 \mathrm{Mar}$ [cited 2020 Apr 14]. https://interagencystandingcommittee. org/system/files/2020-03/IASC\%2OInterim\%20 Briefing \%20Note\%20 on\%20COVID-19\%20 Outbreak\% 20 Readiness $\% 20$ and $\% 20$ Response\%200perations\%20-\%20MHPSS\%20 \%28Portuguese\%29.pdf

13. The British Psychological Society. The psychological needs of healthcare staff as a result of the Coronavirus pandemic: British Psychological Society Covid19, Staff Wellbeing Group [Internet]. 2020 Mar 31 [cited 2020 Apr 14]. www.bps.org. uk/sites/www.bps.org.uk/files/News/News\%20 -\%20Files/Psychological\%20needs\%20of\%20 healthcare\%20staff.pdf

14. Hobfoll SE, Watson P, Bell CC, Bryant RA, Brymer MJ, Friedman MJ, et al. Five essential elements of immediate and mid-term mass trauma intervention: empirical evidence. Psychiatry. 2007;70:283-315; discussion 316-69.

15. Souza EM, Grundy E. Promoção da saúde, epidemiologia social e capital social: inter-relações e perspectivas para a saúde pública. Cad Saude Publica. 2004:20:1354-60.

16. Lee S, Jung M. Social capital, community capacity, and health. Health Care Manag (Frederick). 2018:37:290-8. 
COMUNICAC̣ÃO BREVE

LÍLIAN SCHWANZ LUCAS

ANTÔNIO ALVIN

DEISY MENDES PORTO

ANTÔNIO GERALDO DA SILVA

MAYRA ISABEL CORREIA PINHEIRO
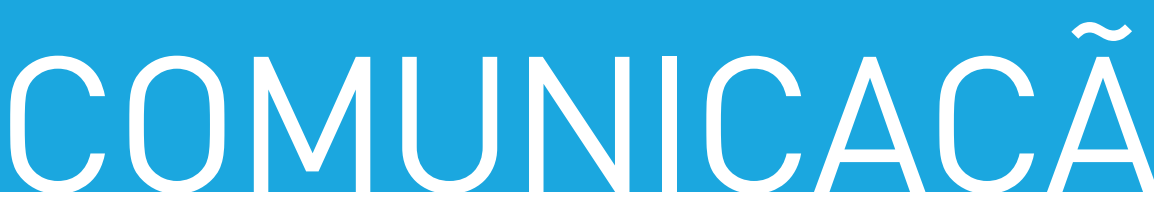

0

\section{IMPACTTOS DA PANDEMIA DE COVID-19 NA SAUDE MENTAL DE CRIANCCAS E ADOLESCENTES: ORIENTACÕEES DO} DEPARTAMENTO DE PSIQUIIATRIA DA INFÂNCIA EADOLESCÊNCIA DA ASSOCIAÇÃO BRASILEIRA DE PSIQUIATRIA

\section{IMPACTS OF THE COVID-19 PANDEMIC ON THE MENTAL HEALTH OF CHILDREN AND ADOLESCENTS: GUIDELINES FROM THE DEPARTMENT OF CHILD AND ADOLESCENT PSYCHIATRY OF THE BRAZILIAN PSYCHIATRIC ASSOCIATION}

\section{Resumo}

A pandemia de coronavírus (COVID-19) é uma emergência de saúde pública de interesse internacional e representa também um desafio à saúde mental, em especial para crianças e adolescentes ainda em desenvolvimento. Para aqueles jovens que já têm uma história de transtornos mentais, os riscos são maiores. Para diminuir as consequências das incertezas da pandemia e do confinamento em casa, governos, comunidade, escola, pais e profissionais de saúde precisam estar informados e abordar de forma efetiva e imediatamente essas questões. $O$ presente artigo tem como objetivo orientar os psiquiatras que precisarão oferecer informações sobre como apoiar e proteger o bem-estar psíquico de crianças e adolescentes.

Palavras-chave: COVID-19, saúde mental, crianças.

\section{Abstract}

The coronavirus (COVID-19) pandemic is a public health emergency of international interest and also represents a challenge to mental health, especially for children and adolescents that are still in development. For those youth who already have a history of mental disorders, the risks are greater. In order to lessen the consequences of the uncertainties associated with the pandemic and with home confinement, governments, communities, schools, parents and mental health professionals need to be informed and address these issues effectively and immediately. This article aims to guide psychiatrists who need to provide information on how to support and protect the psychological well-being of children and adolescents.

Keywords: COVID-19, mental health, children.

Com os casos da pandemia de coronavírus crescendo no mundo, a vida de milhões de crianças e adolescentes foi impactada. A pandemia de coronavírus (coronavirus disease 2019 - COVID-19) é uma emergência de saúde pública de interesse internacional e representa um desafio à resiliência psicológica ${ }^{1}$, em especial dessa população. Em resposta à doença, foi decretado o fechamento de escolas em todo o país como uma medida de emergência para evitar a propagação da infecção, o que atinge cerca de 83,4 milhões de estudantes da educação básica, ensino fundamental e médio ${ }^{2}$. O isolamento da população em suas residências também foi recomendado. 
Vice-coordenadora, Departamento de Psiquiatria da Infância e Adolescência, ABP, Rio de Janeiro, RJ. ${ }^{2}$ Coordenador, Departamento de Psiquiatria da Infância e Adolescência, ABP, Rio de Janeiro, RJ. ${ }^{3}$ Secretária, Departamento de Psiquiatria da Infância e Adolescência, ABP, Rio de Janeiro, RJ. ${ }^{4}$ Universidade de Porto, Porto, Portugal. Associação Brasileira de Psiquiatria (ABP), Rio de Janeiro, RJ. Asociación Psiquiátrica de America Latina (APAL), Brasília, DF. ${ }^{5}$ Secretária, Secretaria de Gestão do Trabalho e da Educação na Saúde do Ministério da Saúde.

Além do Brasil, a maioria dos governos ao redor do mundo fechou temporariamente instituições educacionais, na tentativa de conter a propagação da pandemia de COVID-19. Essa medida afeta, segundo a UNESCO, mais de $91 \%$ da população estudantil do mundo ${ }^{3}$.

Essas ações são necessárias nesse momento para contenção da transmissão de COVID-19 em escala que não seja suportada pelos sistemas de saúde. Tornase relevante, portanto, planejarmos como atenuar os impactos negativos que essas medidas possam ter para a saúde de crianças e adolescentes.

Por outro lado, evidências sugerem que quando as crianças estão fora da escola (por exemplo, nas férias, feriados ou mesmo finais de semana), são fisicamente menos ativas, têm mais tempo de tela, padrões irregulares de sono e dietas menos saudáveis, resultando em ganho de peso e perda de capacidade cardiorrespiratória ${ }^{4-6}$. Além disso, sabemos que a situação causa grande impacto psicológico e que as questões relacionadas à saúde mental de crianças e adolescentes são muitas vezes negligenciadas. Duração prolongada de estresse, temores de infecção, frustração, tédio, informações inadequadas, falta de contato pessoal com colegas de classe, falta de espaço em casa e perdas financeiras na família podem ter ainda efeitos mais duradouros nessa população ${ }^{6}$. E ainda, em algum momento, muitas famílias estarão lidando com o luto de pessoas próximas.

Diversos estudos sugerem que o estresse nas fases iniciais de desenvolvimento pode induzir alterações persistentes na capacidade do eixo hipotálamopituitária-adrenal (HPA) em responder ao estresse na vida adulta, com consequências em longo prazo para a saúde mental global. Em curto e médio prazo, a média dos escores de estresse pós-traumático foi quatro vezes maior em crianças em quarentena?. Nessa equação, a interação entre mudanças de estilo de vida e estresse psicossocial causado por confinamento em casa poderia agravar os efeitos prejudiciais na saúde física e mental da criança, causando um círculo vicioso.

Para diminuir as consequências do confinamento em casa, governos, comunidade, escola e pais precisam estar informados e abordar efetiva e imediatamente essas questões. Experiências aprendidas com surtos anteriores podem ser valiosas nesse momento. Governos precisarão aumentar a conscientização sobre possíveis impactos físicos e mentais na saúde de crianças e adolescentes durante esse período incomum. Para mitigar o impacto imediato do fechamento de escolas, particularmente para comunidades mais vulneráveis e desfavorecidas, e para facilitar a continuidade da educação para todos por meio de aprendizado remoto, será necessário oferecer diretrizes e princípios em aprendizado online eficazes e garantir que o conteúdo dos cursos atenda os requisitos educacionais. No entanto, também é importante não sobrecarregar os estudantes com atividades em excesso nessa fase, o que geraria estresse adicional, que deve ser evitado. Muitos pais também estão sentindo-se sobrecarregados com o trabalho à distância somado a tarefas domésticas e participação em tarefas escolares ${ }^{8}$.

Para adolescentes, é importante lembrar que esse período do desenvolvimento humano pode, por si só, ser de estresse e apreensão. É nessa fase que surge a maior parte dos transtornos mentais, com 50\% de todos os transtornos mentais iniciados até os 14 anos, segundo a Organização Mundial de Saúde (OMS). O cuidado com essa população especial é imperioso. Eles estarão mais vulneráveis mesmo muitos meses depois de cessada essa crise.

A resiliência é o processo humano natural de boa adaptação em face à adversidade. Os indivíduos lidam com o trauma de várias formas e com vários graus de sucesso. As estatísticas nos mostram que a vasta maioria se recupera de experiências traumáticas sem vivenciar psicopatologias significativas, mas para aqueles que desenvolvem problemas clinicamente importantes, a intervenção precoce é a melhor opção. Técnicas que promovem o processamento das informações sobre a vivência traumática e a exposição à informação corretiva são as mais indicadas?.

Durante a fase inicial do surto de COVID-19 na China, mais da metade dos entrevistados do estudo conduzido por Wang et al., realizado através de entrevistas online com moradores de 194 cidades da China, classificou seu impacto psicológico como moderado a grave, e cerca de 1/3 relatou ansiedade moderada a grave. Sexo feminino, status de estudante e sintomas físicos específicos foram associados a um maior impacto psicológico do surto e 
a níveis mais altos de estresse, ansiedade e depressão. Informações adequadas de saúde e certas medidas de precaução foram associadas a um menor impacto psicológico do surto e a níveis mais baixos de estresse, ansiedade e depressão ${ }^{1}$.

Aumento significativo nos níveis de estresse e ansiedade em parte da população é um efeito colateral importante das incertezas da pandemia e do próprio isolamento. Uma metanálise avaliando fatores de risco para transtorno de estresse pós-traumático (TEPT) em crianças e adolescentes de 6 a 18 anos aponta que fatores relacionados à experiência subjetiva do evento (incluindo medo peritraumático e ameaça de vida percebida) e variáveis pós-trauma (incluindo baixo apoio social, retraimento social, comorbidade psiquiátrica, mau funcionamento da família e uso de certas estratégias cognitivas como distração e supressão do pensamento) foram responsáveis pelo tamanho dos efeitos de média tensão na predição do TEPT, enquanto fatores pré-trauma (incluindo sexo feminino, baixa inteligência, baixo status socioeconômico, eventos de vida antes do trauma, baixa autoestima pré-trauma, problemas psicológicos anteriores nos jovens e nos pais) representaram apenas tamanhos de efeito pequenos a médios ${ }^{10}$. Por outro lado, o apoio dos pais, níveis mais baixos de TEPT parental e a resolução de outros sintomas relacionados ao trauma dos pais preveem níveis mais baixos de sintomas de TEPT em crianças.

No contexto de um evento extremo como a pandemia que vivemos, o aumento da visualização, na televisão ou outras telas, de eventos relacionados ao tema, atrasos na evacuação, sintomas de pânico extremos ou sentir que a própria vida ou a de um membro da família está em perigo foram considerados independente e significativamente associados ao desenvolvimento de sintomas de TEPT em crianças ${ }^{11}$. Índices de estresse pós-traumático podem ser iguais aos do terrorismo nessas situações, e crianças são consideradas de maior risco ${ }^{12}$.

Como o sofrimento psicológico moderado e transitório pode ser uma reação normativa à exposição traumática, o TEPT não é diagnosticado até que se tenha passado pelo menos 1 mês desde a ocorrência do evento traumático do índice. Transtorno de estresse agudo ou transtorno de ajustamento podem ser diagnosticados dentro do primeiro mês de exposição ${ }^{11}$.

Em relação à prática clínica, após eventos que podem exceder a capacidade da criança de lidar com o estresse, a primeira necessidadeé, invariavelmente, a informação. O fornecimento desse tipo de suporte pragmático minimiza o estresse adicional. Os profissionais da área de saúde mental proporcionam um apoio importante nessas situações, inclusive na maior difusão possível de informação correta e adequada a crianças através dos meios de comunicação disponíveis. Com relação ao tratamento do transtorno de estresse agudo e TEPT, os resultados mais consistentes são os da terapia cognitivo-comportamental (TCC), em especial as técnicas que envolvem exposição à informação corretiva?.

À medida que as conversas públicas sobre a COVID-19 aumentam, as crianças podem começar a se preocupar que elas mesmas, sua família e seus amigos adoeçam. Pais, familiares, funcionários da escola e outros adultos de confiança podem desempenhar um papel importante ao ajudar as crianças a entenderem o que ouvem de maneira honesta e precisa e minimizarem a ansiedade ou o medo. Assim, é importante mostrar-se disposto a ouvir e conversar, permanecer calmo e reconfortante, lembrando que as crianças reagirão ao que você diz e como diz.

Usar linguagem própria à etapa de desenvolvimento da criança é fundamental. Manter, na medida do possível, a rotina em casa e buscar atividades com propósito ajuda a manter o senso de continuidade e de que o que estamos vivendo é transitório e vai passar. É preciso evitar linguagem que possa culpar os outros e levar ao estigma. Lembre-se de que os vírus podem deixar qualquer pessoa doente, independentemente da sua raça ou etnia. Evite fazer suposições sobre quem pode ter a COVID-19. A exposição às notícias sobre a pandemia deve ser reduzida, pois pode ser exaustiva para crianças e nem sempre traz informações acuradas ${ }^{13}$.

Por outro lado, este é um momento extremo, e a flexibilidade em relação a normas rígidas demais em relação ao uso de internet, por exemplo, é necessária. Crianças e adolescentes da geração digital podem estar usando suas particularidades como fator de resiliência 
1 Vice-coordenadora, Departamento de Psiquiatria da Infância e Adolescência, ABP. Rio de Janeiro, RJ. ${ }^{2}$ Coordenador, Departamento de Psiquiatria da Infância e Adolescência, ABP, Rio de Janeiro, RJ. ${ }^{3}$ Secretária, Departamento de Psiquiatria da Infância e Adolescência, ABP, Rio de Janeiro, RJ. ${ }^{4}$ Universidade de Porto, Porto, Portugal. Associação Brasileira de Psiquiatria (ABP). Rio de Janeiro, RJ. Asociación Psiquiátrica de America Latina (APAL), Brasília, DF. ${ }^{5}$ Secretária, Secretaria de Gestão do Trabalho e da Educação na Saúde do Ministério da Saúde.

diante das dificuldades experimentadas agora. Com mais facilidade, podem adaptar-se ao uso de recursos de aprendizagem online, por exemplo. $\bigcirc$ uso desses recursos pelos jovens, ainda bastante questionado em muitas esferas, em poucos dias tornou-se fundamental no enfrentamento da crise, ajudando a prover soluções para os problemas que surgem na comunicação, no enfrentamento ao isolamento, na difusão de informações sobre a pandemia e no ensino. Manter essa população ativa, praticando exercícios físicos possíveis, é também importante.

Temos um sistema de assistência em saúde mental ainda precário para o atendimento dessa população. Assim, investimentos e soluções criativas, como modalidades de atendimento em rede, serão cruciais na administração da crise e pós-crise. A Associação Americana de Psiquiatria da Infância e Adolescência $(A A C A P)^{11}$ recomenda, por exemplo, realizar rastreamento escolar ou na comunidade para detectar sintomas e fatores de risco de TEPT após eventos traumáticos que afetam um número significativo de crianças.

A saúde mental faz parte da missão de lidar com doenças transmissíveis ${ }^{13}$. Assim, é fundamental que as considerações sobre saúde mental se tornem parte integrante da resposta à pandemia. Não poderemos perder de vista as enormes consequências dessa ameaça à saúde e ao desenvolvimento das nossas crianças mais vulneráveis e das suas famílias - agora e nos próximos anos. Psiquiatras precisarão oferecer informações sobre como apoiar e proteger o bem-estar emocional, à medida que a crise de saúde pública se desenrola.

Artigo submetido em 28/04/2020, aceito em 28/04/2020. Os autores informam não haver conflitos de interesse associados à publicação deste artigo.

Fontes de financiamento inexistentes.

Correspondência: Lílian Schwanz Lucas, Av. Rio Branco, 404, torre 2, S 806, CEP 88015-200, Florianópolis, SC. E-mail: I_slucas@hotmail.com

\section{Referências}

1. Wang C, Pan R, Wan X, Tan Y, Xu L, Ho CS, et al. Immediate psychological responses and associated factors during the initial stage of the 2019 coronavirus disease (COVID-19) Epidemic among the General Population in China. Int J Environ Res Public Health. 2020 Mar 6;17(5). pii: E1729. doi: 10.3390/ijerph17051729.

2. Brasil, Ministério da Educação, Instituto Nacional de Estudos e Pesquisas Educacionais Anísio Teixeira (INEP). Censo escolar [Internet]. 2015 Oct 20 [cited 2020 Apr 29]. http:/ www.portal. inep.gov.br/censo-escolar

3. United Nations Educational Scientific and Cultural Organization (UNESCO). Educational disruption and response [internet]. [cited 2020 Mar 31]. https://en.unesco.org/covid19/ educationresponse

4. Wang G, Zang Y, Zhao J, Zhang J, Jiang F. Mitigate the effects of home confinement on children during the COVID-19 outbreak. Lancet. 2020;395:945-7.

5. Brazendale K, Beets MW, Weaver RG, Pate RR, Turner-McGrievy GM, Kaczynski AT, et al. Understanding differences between summer vs. school obesogenic behaviors of children: the structured days hypothesis. Int J Behav Nutr Phys Act. 2017;14:100.

6. Brooks SK, Webster RK, Smith LE, Woodland L, Wessely S, Greenberg N, et al. The psychological impact of quarantine and how to reduce it: rapid review of the evidence. Lancet. 2020;395:91220.

7. Sprang G, Silman M. Posttraumatic stress disorder in parents and youth after health- related disasters. Disaster Med Public Health Prep. 2013;7:105-10.

8. Decosimo CA, Hanson J, Quinn M, Badu P, Smith EG. Playing to live: outcome evaluation of a community-based psychosocial expressive arts program for children during the Liberian Ebola epidemic. Glob Ment Health (Camb). 2019;6:e3.

9. Devilly GJ, Wright R, Gist R. [The role of psychological debriefing in the treatment of victims of trauma]. Braz J Psychiatry. 2003;25 Supll 1:41-5.

10. Bui E, Ohye B, Palitz S, Olliac B, Goutaudier N, Raynaud JP, et al. Acute and chronic reactions to trauma in children and adolescents [Internet]. 


\section{COMUNICAÇÃO BREVE}

\section{LÍLIAN SCHWANZ LUCAS}

ANTÔNIO ALVIN

DEISY MENDES PORTO

ANTÔNIO GERALDO DA SILVA MAYRA ISABEL CORREIA PINHEIRO

[cited 2020 Apr 29]. https://iacapap.org/content/ uploads/F4-PTSD-2014.pdf

11. Cohen JA, Bukstein $O$, Walter $H$, Benson SR, Chrisman A, Farchione TR, etal. Practice parameter for the assessment and treatment of children and adolescents with posttraumatic stress disorder. J Am Acad Child Adolesc Psychiatry. 2010;49:41430.
12. Brock SE, Lazarus PJ Jr, Jimerson SR. Best practices in school crisis prevention and intervention. Bethesda: NASP; 2002.

13. Hamoda H. "Remember": surviving the pandemic with your children! [Internet]. [cited 2020 Apr 7]. http://iacapap.org/remember-surviving-thepandemic-with-your-children

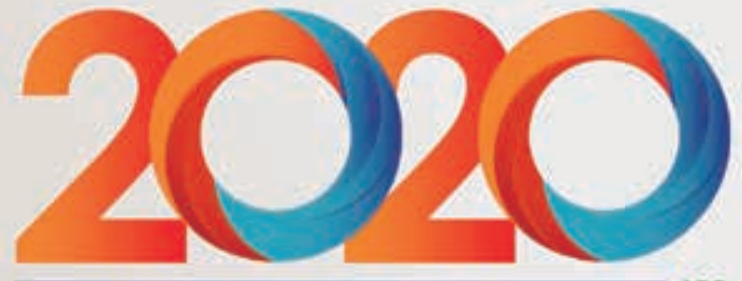

ANUIDADE DA ABP

FORTALEÇA $\mathrm{H}$ SUA ESPECIALIDADE!

Há 53 anos fazendo 0 melhor pela psiquiatria brasileira. Fique em dia!
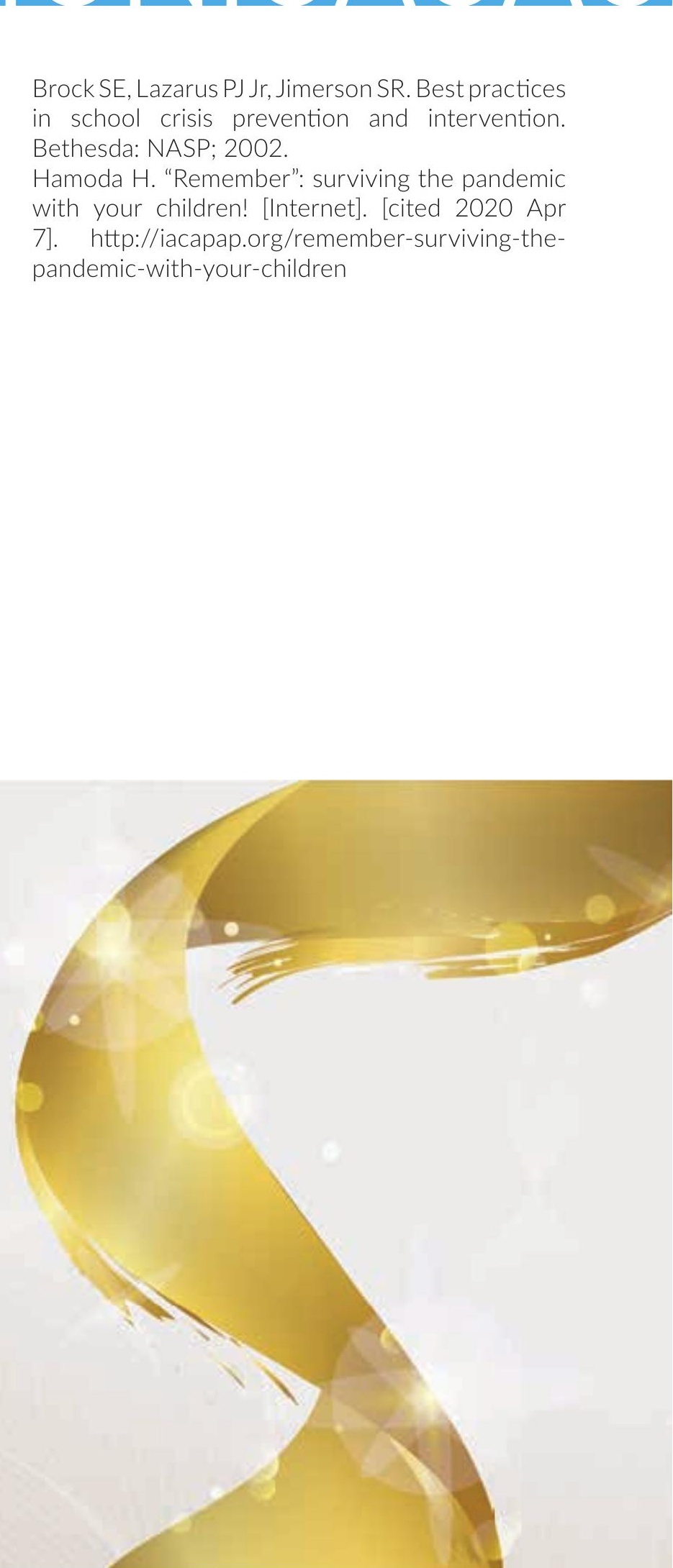